\title{
CFBDS J111807-064016: A new L/T transition brown dwarf in a binary system ${ }^{\star}$
}

\author{
C. Reylé ${ }^{1}$, P. Delorme ${ }^{2}$, E. Artigau ${ }^{3}$, X. Delfosse ${ }^{2}$, L. Albert ${ }^{3}$, T. Forveille ${ }^{2}$, A. S. Rajpurohit ${ }^{1}$, \\ F. Allard ${ }^{4}$, D. Homeier ${ }^{4}$, and A. C. Robin ${ }^{1}$ \\ 1 Institut UTINAM, CNRS UMR 6213, Observatoire des Sciences de l'Univers THETA Franche-Comté-Bourgogne, \\ Université de Franche Comté, Observatoire de Besançon, BP 1615, 25010 Besançon Cedex, France \\ e-mail: celine@obs-besancon.fr \\ 2 UJF-Grenoble 1/CNRS-INSU, Institut de Planétologie et d'Astrophysique de Grenoble (IPAG) UMR 5274, 38041 Grenoble, France \\ 3 Département de physique and Observatoire du Mont Mégantic, Université de Montréal, CP 6128, Succursale Centre-Ville, \\ Montréal, QC H3C 3J7, Canada \\ ${ }^{4}$ Centre de Recherche Astrophysique de Lyon, CNRS UMR 5574, Université de Lyon, École Normale Supérieure de Lyon, \\ 46 allée d'Italie, 69364 Lyon Cedex 7, France
}

Received 19 June 2013 / Accepted 4 September 2013

\begin{abstract}
Binary systems with a substellar companion are quite rare and provide interesting benchmarks. They constrain the complex physics of substellar atmospheres, because several physical parameters of the substellar secondary can be fixed from the much better characterized main-sequence primary. We report the discovery of CFBDS J111807-064016, a T2 brown-dwarf companion to 2MASS J111806.99-064007.8, a low-mass M4.5-M5 star. The brown dwarf was identified from the Canada France Brown Dwarf Survey. At a distance of 50-120 pc, the 7.7" angular separation corresponds to projected separations of 390-900 AU. The primary displays no $\mathrm{H}_{\alpha}$ emission, placing a lower limit on the age of the system of about 6 Gyr. The kinematics is also consistent with membership in the old thin disc. We obtained near-infrared spectra, which together with recent atmosphere models allow us to determine the effective temperature and gravity of the two components. We derived a system metallicity of $[\mathrm{Fe} / \mathrm{H}]=-0.1 \pm 0.1 \mathrm{using}$ metallicity-sensitive absorption features in our medium-resolution $K_{\mathrm{s}}$ spectrum of the primary. From these parameters and the age constraint, evolutionary models estimate masses of 0.10 to $0.15 M_{\odot}$ for the $\mathrm{M}$ dwarf and 0.06 to $0.07 M_{\odot}$ for the T dwarf. This system is a particularly valuable benchmark because the brown dwarf belongs to the early-T class: the cloud-clearing that occurs at the $\mathrm{L} / \mathrm{T}$ transition is very sensitive to gravity, metallicity, and detailed dust properties, and produces a large scatter in the colours. This T2 dwarf, with its metallicity measured from the primary and its mass and gravity much better constrained than those of younger early-Ts, will anchor our understanding of the colours of L/T transition brown dwarfs. It is also one of the most massive T dwarfs, just below the hydrogen-burning limit, and all this makes it a prime probe for brown-dwarf atmosphere and evolution models.
\end{abstract}

Key words. stars: individual: CFBDS J111807-064016 - stars: individual: 2MASS J111806.99-064007.8 - stars: low-mass brown dwarfs - binaries: general

\section{Introduction}

Observational degeneracies between the influences of age, metallicity, and effective temperature hinder our understanding of the physics of atmospheres in the brown-dwarf and exoplanetary temperature range. This leads to ambiguities in mapping observational measurements to physical parameters (effective

\footnotetext{
* Based on observations obtained with MegaPrime/MegaCam, a joint project of CFHT and CEA/DAPNIA, at the Canada-France-Hawaii Telescope (CFHT) which is operated by the National Research Council (NRC) of Canada, the Institut National des Sciences de l'Univers of the Centre National de la Recherche Scientifique (CNRS) of France, and the University of Hawaii. This work is based in part on data products produced at TERAPIX and the Canadian Astronomy Data Centre as part of the Canada-France-Hawaii Telescope Legacy Survey, a collaborative project of NRC and CNRS. Based on observations made with the ESO New Technology Telescope at the La Silla Observatory under programme ID 082.C-0506(A) and the ESO Very Large Telescope at Paranal Observatory under programme ID 385.C-0242(A). Based on observations (director discretionary time) made with the CanadaFrance-Hawaii Telescope at Mauna Kea Observatory.
}

temperature, mass, gravity, metallicity) using theoretical models. These are also much less reliable than models for stellar objects, in part because the physics of their clouds challenges our understanding.

Brown-dwarfs main-sequence-star binaries uniquely break most of this degeneracy, because several parameters can be obtained from the better-understood primary. Such benchmarks systems are rare, however. When the two components are sufficiently separated to be studied separately, simple spectroscopic observations of the bright primary measure the metallicity of the system and constrain its age. This greatly helps in interpreting the spectrum of the brown dwarf by fixing its age and composition. The photometric distance of the primary - and thus the luminosity of both components - is also less uncertain, and followup parallax measurements are more easily carried out. Saumon et al. (2006), studying the atmosphere of Gl 570 D, found the first evidence for departures from local chemical equilibrium in the high atmosphere of $\mathrm{T}$ dwarfs from investigating the kinetics of nitrogen and carbon chemistry in the presence of vertical mixing. Leggett et al. (2008) showed that the models of that 
Table 1. Known binary systems with a main-sequence primary and a T-dwarf secondary.

\begin{tabular}{|c|c|c|c|c|c|c|}
\hline \multirow[t]{2}{*}{ Name } & \multicolumn{2}{|c|}{ Sp. Type } & \multirow{2}{*}{$\begin{array}{l}\text { Dist. } \\
\text { (pc) }\end{array}$} & \multicolumn{2}{|c|}{ Sep. } & \multirow[t]{2}{*}{ Ref. } \\
\hline & Primary & Secondary & & $(\mathrm{AU})$ & $\left({ }^{\prime \prime}\right)$ & \\
\hline G1 $337 \mathrm{CD}$ & $\mathrm{K} 1$ & L8/T0 & 20.5 & 881 & 43 & Wilson et al. (2001); Burgasser et al. (2005) \\
\hline HD 46588 B & F7 & L9 & 17.9 & 1420 & 79.2 & Loutrel et al. (2011) \\
\hline$\epsilon$ Ind $\mathrm{Ba}$ & K5 & $\mathrm{T} 1$ & 3.6 & 1459 & 405 & Scholz et al. (2003) \\
\hline CFBDS 1118 & M4.5 & $\mathrm{T} 2$ & 92 & 709 & 7.7 & This paper \\
\hline HN Peg B & G0V & $\mathrm{T} 2.5$ & 18.4 & 795 & 43.2 & Luhman et al. (2007) \\
\hline HIP 38939 B & K4 & T4.5 & 18.5 & 1630 & 88 & Deacon et al. (2012b) \\
\hline 2MASS J03202839-0446358 ${ }^{a}$ & M8.5 & T5 & 25 & $<8.3$ & $<0.33$ & Burgasser et al. (2008); Blake et al. (2008) \\
\hline SDSS J000649.16-085246.3 B ${ }^{b}$ & M8.5 & T5 & 30 & 0.286 & 0.0095 & Burgasser et al. (2012) \\
\hline HD 118865 B & F5 & T5 & 62.4 & 9200 & 148 & Burningham et al. (2013) \\
\hline LHS 2803 B & M4.5 & T5.5 & 21 & 1400 & 67.6 & Deacon et al. (2012a) \\
\hline$\epsilon$ Ind $\mathrm{Bb}$ & K5 & T6 & 3.6 & 1459 & 405 & Scholz et al. (2003) \\
\hline SCR 1845 B & M8.5 & T6 & 3.8 & 4.5 & 1.2 & Biller et al. (2006) \\
\hline HIP 73786 B & K5 & T6.5 & 18.6 & 1260 & 68 & Scholz (2010) \\
\hline G 204-39 & M3 & T6.5 & 13.6 & 2685 & 198 & Faherty et al. (2010) \\
\hline G1 229 B & M1 & $\mathrm{T} 7$ & 5.8 & 45 & 7.8 & Nakajima et al. (1995) \\
\hline HIP $63510 \mathrm{C}$ & M0.5 & $\mathrm{T} 7$ & 11.7 & 1200 & 103 & Scholz (2010) \\
\hline Gl $570 \mathrm{D}$ & K4 & $\mathrm{T} 7.5$ & 5.9 & 1525 & 258.3 & Burgasser et al. (2000) \\
\hline HD $3651 \mathrm{~B}$ & K0 & $\mathrm{T} 7.5$ & 11 & 480 & 43.5 & Mugrauer et al. (2006); Luhman et al. (2007) \\
\hline Ross $458 \mathrm{C}$ & M2 & $\mathrm{T} 8$ & 11.4 & 1163 & 102 & Goldman et al. (2010); Burgasser et al. (2010) \\
\hline LHS $6176 \mathrm{~B}$ & M4 & $\mathrm{T} 8$ & 18.7 & 970 & 52 & Burningham et al. (2013) \\
\hline Wolf $940 \mathrm{~B}$ & M4 & T8.5 & 12.5 & 400 & 32 & Burningham et al. (2009) \\
\hline GJ $758 \mathrm{~B}$ & G9 & T9 & 15.5 & 29 & 1.9 & Thalmann et al. (2009) \\
\hline
\end{tabular}

Notes. The listed physical separations are the projected separations. The last column is the reference of the discovery paper. ${ }^{(a)}$ Unresolved system; (b) unresolved system, separation is estimated from the radial velocity orbit.

time were only able to fit $\mathrm{HN}$ Peg B at the minimum admissible age for the HN Peg system and simultaneously required significant vertical atmospheric mixing. Burningham et al. (2009) used the Wolf 940 system to conclude that model-derived temperatures of very late T dwarfs are $10 \%$ too warm. Detailed studies of T-dwarf companions to main-sequence stars therefore constrain our understanding of $\mathrm{T}$ dwarfs in general. Moreover, the frequencies with which these systems occur and the distribution functions of their orbital parameters constrain theories for brown-dwarf formation (Reipurth \& Clarke 2001; Bate et al. 2003; Stamatellos \& Whitworth 2009).

To date, twenty resolved T-dwarf main-sequence-star systems are confirmed (Table 1). These rare systems constrain coolatmosphere physics regardless of the brown-dwarf spectral type, but they are most valuable in temperature ranges that are poorly described by models. Our understanding of T-dwarf atmospheres is most highly challenged at the L-T transition, where cloudclearing processes dramatically change the shape of the emerging spectrum. While cool-atmosphere models perform relatively well in fully dusty atmospheres (late-M to late-L), in fully dustfree atmospheres (early-T) and in the mid-T dwarfs where lowtemperature condensates appear (Morley et al. 2012), they currently describe the more complex physics at the $\mathrm{L} / \mathrm{T}$ transition only poorly (e.g. Allard et al. 2001; Helling et al. 2008).

The transition is observationally characterized by the nearinfrared colours swinging from very red in the late- $\mathrm{L}$ and early$\mathrm{T}$ dwarfs to very blue in the mid-T dwarfs. This change occurs over a narrow effective temperature range, $\sim 1100$ to $1400 \mathrm{~K}$ (Kirkpatrick et al. 2000; Golimowski et al. 2004; Vrba et al. 2004; Dupuy \& Liu 2012). The detection of variablity in a few of these objects (e.g. Artigau et al. 2009; Radigan et al. 2012) suggests, moreover, that cloud coverage is spatially inhomogeneous and additionally complicates the modelling and interpretation.
This transition is currently poorly constrained observationally. Of the 20 resolved (in seeing-limited observations) binaries that consist of a $\mathrm{T}$ dwarf and a main-sequence star, only five (G1337 D, HD 46588 B, HN Peg B, $\epsilon$ Ind Ba, and our discovery) have an early- $\mathrm{T}$ secondary, the others harbour mid-to-late T companions.

We report here the discovery of CFBDS J111807-064016 (hereafter CFBDS 1118), an early-T dwarf bound to a mid-M dwarf, 2MASS J111806.99-064007.8 (hereafter 2MASS 1118). We found the brown dwarf in the Canada France Brown Dwarf Survey (CFBDS), a wide-field survey for cool brown dwarfs that we conducted with the MegaCam camera (Boulade et al. 2003) on the Canada France Hawaii Telescope (CFHT), and subsequently identified the primary from its common proper motion. In Sect. 2 we describe our identification of the system and its near-infrared photometric and spectroscopic followup. Section 3 examines the physical properties of the system. Section 4 discusses the physical properties of the $T$ dwarf and contrasts them with those of HN Peg B, a T2.5 dwarf with very different age and mass. The last section summarizes our conclusions.

\section{Observations}

We first identified CFBDS 1118 as a brown-dwarf candidate from its red $i^{\prime}-z^{\prime}$ colour in the CFBDS. The aims of the survey and its detection techniques are fully described in Delorme et al. (2008b). CFBDS 1118 is undetected in $i^{\prime}$, with a $5 \sigma i^{\prime}>24.8$ upper limit), but strongly detected at $z^{\prime}\left(z^{\prime}=22.56 \pm 0.05\right)$. The $i^{\prime}-z^{\prime}>2.3(5 \sigma)$ lower limit made CFBDS 1118 a strong brown-dwarf candidate.

Figure 1 shows a $J$-band image of the brown dwarf, which lies 7.7" away from a much brighter star identified as 2MASS 1118 (in the 2MASS survey, Skrutskie et al. 2006). 
C. Reylé et al.: CFBDS 1118: a new L/T transition brown dwarf in a binary system

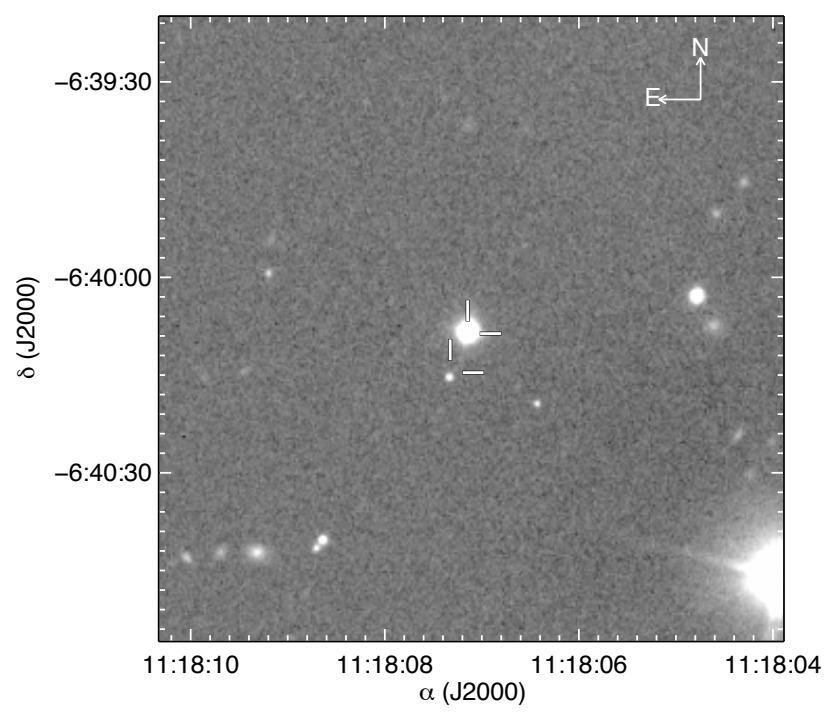

Fig. 1. $J$-band image obtained with WIRCam at CFHT. The tick marks identify the two components 2MASS 1118 (brighter) and CFBDS 1118 (fainter).

Table 2. Positions, proper motions, and photometry of the two components.

\begin{tabular}{lcc}
\hline \hline & M dwarf & T dwarf \\
\hline$\alpha$ & $11 \mathrm{~h} 18 \mathrm{~m} 06.99 \mathrm{~s}$ & $11 \mathrm{~h} 18 \mathrm{~m} 07.13 \mathrm{~s}$ \\
$\delta$ & $-06^{\circ} 40^{\prime} 07.84^{\prime \prime}$ & $-06^{\circ} 40^{\prime} 15.82^{\prime \prime}$ \\
$i^{\prime}$ & & $>24.86$ \\
$z^{\prime}$ & & $22.56 \pm 0.08$ \\
$J$ & $13.84 \pm 0.03$ & $19.01 \pm 0.02$ \\
$H$ & $13.25 \pm 0.02$ & $18.58 \pm 0.03$ \\
$K_{s}$ & $12.95 \pm 0.03$ & $18.30 \pm 0.03$ \\
$\mu_{\alpha}$ & $-201 \pm 11 \mathrm{mas} / \mathrm{yr}$ & $-190 \pm 14 \mathrm{mas} \mathrm{yr}^{-1}$ \\
$\mu_{\delta}$ & $-49 \pm 20 \mathrm{mas} / \mathrm{yr}$ & $-60 \pm 21 \mathrm{mas} \mathrm{yr}^{-1}$ \\
\hline
\end{tabular}

Notes. The photometry of the M dwarf is taken from 2MASS, and the near-infrared magnitudes of the T dwarf are given in the 2MASS system. The $z^{\prime}$ magnitude is given in the AB system used by CFHT for $\mathrm{MegaCam}^{2}$. The proper motion of the T dwarf is computed between the first and third observation epoch. The proper motions are relative to the mean motion of the stars in the field.

The astrometry, proper motions, and photometry ${ }^{1}$ of the components are summarized in Table 2.

\subsection{Near-infrared photometry}

We obtained near-infrared follow-up in the $J, H$, and $K_{\mathrm{s}}$ bands in the 2MASS system. Our $J$-band and $H$-band imaging consists of twenty 30 -second and thirty 20 -second long dithered exposures with the SOFI (Moorwood et al. 1998) near-infrared camera on the New Technology Telescope (NTT) at the European Southern Observatory (ESO), La Silla, on March 7, 2009 (Program 082.C-0506(A)). We used a modified version of the jitter utility within the ESO Eclipse package (Devillard 1997) to correct for the flat field, subtract the background, and coadd the exposures. We also obtained $K_{\mathrm{s}}$-band observations of CFBDS 1118 with WIRCam (Puget et al. 2004) at CFHT on March 12, 2012.

\footnotetext{
1 Throughout this paper, we use Vega magnitudes for the near-infrared bands and $A B$ magnitudes for the $i^{\prime}$ and $z^{\prime}$ optical bands.

2 http://www.cfht.hawaii.edu/Instruments/Imaging/ MegaPrime/specsinformation.html
}

Table 3. Summary of our imaging observations of CFBDS 1118.

\begin{tabular}{lccc}
\hline \hline Filter & Camera & Exposures & Date \\
\hline$z^{\prime}$ & CFHT-MegaCam & $1 \times 360 \mathrm{~s}$ & 12 April 2007 \\
$i^{\prime}$ & CFHT-MegaCam & $1 \times 500 \mathrm{~s}$ & 5 January 2008 \\
$J$ & NTT-SOFI & $20 \times 30 \mathrm{~s}$ & 5 March 2009 \\
$H$ & NTT-SOFI & $31 \times 20 \mathrm{~s}$ & 7 March 2009 \\
$K_{\mathrm{s}}$ & CFHT-WIRCam & $17 \times 21 \mathrm{~s}$ & 12 March 2012 \\
$J$ & CFHT-WIRCam & $5 \times 26 \mathrm{~s}$ & 5 March 2012 \\
\hline
\end{tabular}

That sequence consists of 2120 -s-dithered exposures. Table 3 summarizes our imaging observations.

We extracted photometry from the resulting images using point-spread-function fitting within Source Extractor (Bertin \& Arnouts 1996) and obtained $J=19.01 \pm 0.02, H=18.58 \pm 0.03$, and $K_{\mathrm{s}}=18.30 \pm 0.03$. The resulting $z^{\prime}-J=3.55 \pm 0.08$, $J-H=0.43 \pm 0.03$, and $H-K_{\mathrm{s}}=0.38 \pm 0.04$ colours confirmed CFBDS 1118 as a brown dwarf at the L/T transition (see e.g. Figs. 1 in Reylé et al. 2010; Delorme et al. 2012). In addition to confirming CFBDS 1118 as a brown dwarf, the photometry was used to flux-calibrate the spectrum (Sect. 2.4).

\subsection{Proper motions}

The apparent magnitudes $(J=19.01$ and $J=13.84)$ and colours of the $\mathrm{M}$ and $\mathrm{T}$ dwarfs place them in a common distance range of 70 to $120 \mathrm{pc}$ from Earth (Sect. 3.1). Comparison of their proper motions also probes whether they form a physical binary.

We first computed the proper motion of the T dwarf from its positions in the $z^{\prime}$-band image (observed on April 12, 2007) and in the $J$-band image (observed on March 07, 2009). That value agreed within $1-\sigma$ with the proper motion listed in the NOMAD Catalog (Zacharias et al. 2004) for the M dwarf, but was too noisy $\left(\sigma_{\mu_{\alpha}}=64\right.$ mas $\left.\mathrm{yr}^{-1}, \sigma_{\mu_{\delta}}=97 \mathrm{mas}_{\mathrm{yr}}{ }^{-1}\right)$ to strongly exclude a chance alignement.

We therefore obtained a third-epoch observation in the $J$ band with WIRCam at CFHT on March 5, 2012. We did not attempt to reference the astrometry to an absolute frame, as experience shows that this results in larger errorbars, and instead calculated a relative local astrometric solution. This was achieved by cross-matching the epochs and using Scamp (Bertin 2006) to reference all astrometric solutions to the first-epoch image. Adding the third epoch reduces the error bars on the $\mathrm{T}$ dwarf proper motion measurements by a factor of 5 and allows a much stronger test for chance alignment. Table 2 lists these improved measurements.

\subsection{Binarity characterization}

As Table 2 shows, the proper motions agree to within $1 \sigma$. Simulations of the Galactic population with the Besançon Galaxy model (Robin et al. 2003) in the direction of CFBDS 1118 show an only $\sim 3 \times 10^{-5}$ probability that any mainsequence star with a proper motion within $3 \sigma$ of the T dwarf lies by chance in the volume of space delimited by a cone section of $8^{\prime \prime}$ aperture and $120 \mathrm{pc}$ depth in the direction of CFBDS 1118. Since the CFBDS survey found $\sim 70 \mathrm{~T}$ dwarfs in CFBDS, the probability that at least one of them would so closely align by chance with an unrelated main-sequence star is just $\sim 2 \times 10^{-3}$. Even one chance alignment within the full survey scale is thus very unlikely, and from consequently we discuss CFBDS 1118 and 2MASS 1118 as a physical binary system. 


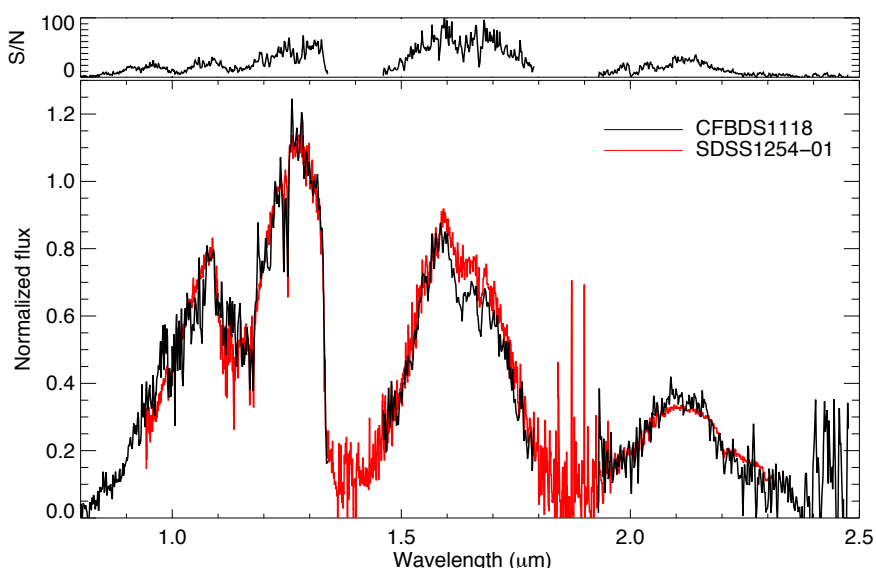

Fig. 2. Black: spectrum of CFBDS 1118 obtained with $\mathrm{X}$-shooter at VLT. The regions with strong telluric $\mathrm{H}_{2} \mathrm{O}$ absorptions cannot be used and are blanked out. Red: T2 dwarf, SDSS J125453.9-012247 (McLean et al. 2007). A scaling factor is applied to normalize the median flux in the range $1.2-1.3 \mu \mathrm{m}$ to unity. The upper panel shows the signal-tonoise ratio.

\subsection{Spectroscopic follow-up}

We obtained near-infrared spectra of CFBDS 1118 with the $\mathrm{X}$-shooter spectrograph (Vernet et al. 2011) on the Very Large Telescope (VLT-UT2) at ESO through program 385.C-0242(A). The observations were carried out as three 1-hour observing blocks on April 13, 2011, achieving a total exposure time on target of 132 min, split into six on-the-slit A-B nods of $2 \times 660 \mathrm{~s}$ each. The slit width was $1.2^{\prime \prime}$, and the seeing varied in the range $0.7-0.9^{\prime \prime}$. We chose a wide slit at some cost in increased sky background, because the T dwarf is not visible on the acquisition camera and was set on the slit through a blind offset from the primary. The spectra for individual observing blocks were reduced using the standard ESO X-shooter pipeline (Modigliani et al. 2010), which produces a 2D, curvature-corrected spectrum. We used our own IDL procedures to extract the trace, using Gaussian boxes in the spatial dimension at each wavelength. A similar Gaussian extraction box 5 FWHM off the trace was used to obtain the spectrum of the sky. The resulting 1D spectrum from each science target observing block was then divided by the spectra of reference stars observed just before or after to remove the telluric absorptions. These were reduced and extracted using the same pipelines. The resulting spectra from the three observing blocks were then median-combined into a final science spectrum. Since that spectrum (with $R=\lambda / \Delta \lambda \simeq 3900$ ) has a low signal-to-noise ratio, we smoothed the spectra using a weighted average over 100 pixels in the wavelength dimension in the visible arm and over 20 pixels in the near-infrared arm. Our weighting is by the inverse variance of the extracted sky spectrum and therefore uses the full spectral resolution of $\mathrm{X}$-shooter to downweight wavelengths affected by telluric emission lines. This improves the signal-to-noise ratio significantly over simple binning or observations with lower resolution. The final resolution is $R \simeq 1100$ and the resulting signal-to-noise ratio is shown in Fig. 2 (upper panel).

The same reduction and extraction procedures were used for the near-infrared and visible arms of X-shooter. Given the wide wavelength range covered by X-shooter, from $0.6 \mu \mathrm{m}$ to $2.5 \mu \mathrm{m}$, seeing variation along the spectral direction can produce wavelength-dependent slit losses. We ensured the flux homogeneity of this wide wavelength spectrum by calibrating it onto our NTT and WIRCam photometry in the $J, H$, and $K_{\mathrm{s}}$ band

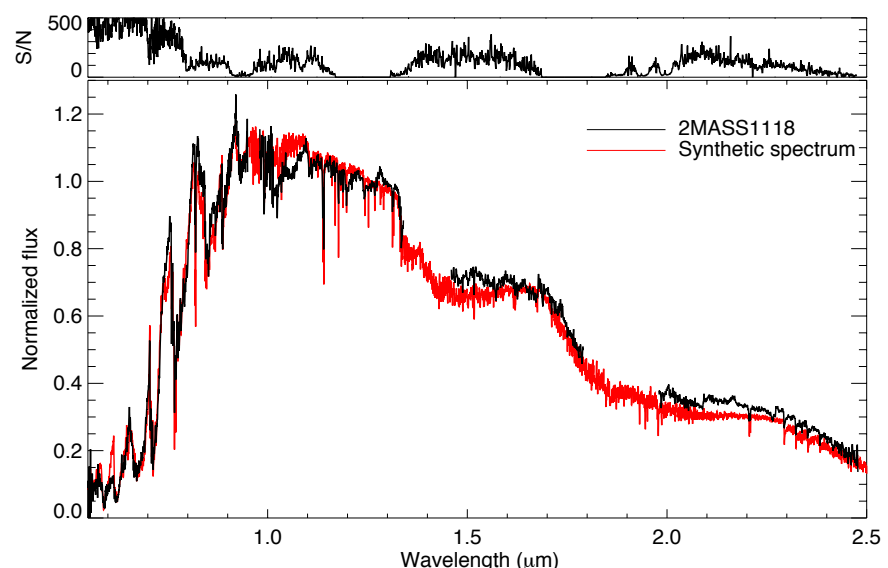

Fig. 3. Spectrum of 2MASS 1118 obtained with X-shooter at VLT in the visible $\operatorname{arm}(\lambda<1 \mu \mathrm{m})$ and the near-infrared arm $(\lambda>1 \mu \mathrm{m})$. The regions with strong telluric $\mathrm{H}_{2} \mathrm{O}$ absorptions cannot be used and are blanked out. The red line shows the synthetic spectrum fitted to derive the astrophysical parameters (see Sect. 3). A scaling factor is applied to normalize the median flux in the range $1.2-1.3 \mu \mathrm{m}$ to unity. The upper panel shows the signal-to-noise ratio.

(Table 2) and onto a near-infrared $Y$-band magnitude that we estimated from the average colours of a T2 dwarf (Burningham et al. 2013). Following the procedure described in Delorme et al. (2010), we applied small scaling factors to each broadband wavelength ranges to match synthetic photometry derived from the CFBDS 1118 spectrum to its observed photometry. The final spectrum is shown in Fig. 2.

We also observed the primary star with X-shooter. The $\mathrm{M}$ dwarf is detected with a high signal-to-noise ratio in all $\mathrm{X}$-shooter arms (see upper panel in Fig. 3) and its spectrum was extracted following the procedures described for the $\mathrm{T}$ dwarf. The final spectrum, smoothed using a weighted average over ten pixels in the wavelength dimension, is shown in Fig. 3.

\section{Physical properties}

\subsection{Spectral type}

We computed spectral indices and derived a spectral type for both components. For the M dwarf, we used the classification scheme defined by Reid et al. (1995) based on $\mathrm{TiO}$ and $\mathrm{CaH}$ bandstrengths. For the T dwarf, we computed the spectroscopic indices defined by Burgasser et al. (2006) and Delorme et al. (2008a), which quantify the strength of the key molecular absorption bands, $\mathrm{H}_{2} \mathrm{O}$ and $\mathrm{CH}_{4}$. Table 4 lists these spectral indices and the corresponding spectral types. This classifies the primary as an M4.5 to M5 dwarf and CFBDS 1118 as a T2 to T3 dwarf. Figure 2 shows the agreement between the spectra of CFBDS 1118 and SDSS J125453.9-012247, a T2 spectral type standard from the NIRSPEC Brown Dwarf Spectroscopic Survey (McLean et al. 2007).

\subsection{Photometric distance and separation}

To compute the photometric distance of 2MASS 1118, we used several $M_{J}$ versus spectral type relations from the literature, as described in Reylé et al. (2006). The $M_{J}$ absolute magnitude of an M4.5 to M5 dwarf ranges from 8.50 to 9.72 , taking into account the uncertainties in the relation, and in particular the discontinuity in the relation at $M_{J} \simeq 8.5$ (or spectral 
Table 4. Spectral indices and corresponding spectral types of the two components.

\begin{tabular}{lcc}
\hline \hline & M dwarf \\
\hline TiO 5 & 0.265 & M5 \\
$\mathrm{CaH} \mathrm{1}$ & 0.707 & M4.5 \\
$\mathrm{CaH} 2$ & 0.347 & M4.5 \\
$\mathrm{CaH} \mathrm{3}$ & 0.638 & M5 \\
\hline & T dwarf \\
\hline $\mathrm{H}_{2} \mathrm{O}-\mathrm{J}$ & 0.472 & T2 \\
$\mathrm{CH}_{4}-\mathrm{J}$ & 0.603 & T2 \\
$\mathrm{H}_{2} \mathrm{O}-\mathrm{H}$ & 0.482 & T2 \\
$\mathrm{CH}_{4}-\mathrm{H}$ & 0.815 & T2 \\
$\mathrm{CH}_{4}-\mathrm{K}$ & 0.400 & T3 \\
\hline
\end{tabular}

Table 5. Fraction of stars as a function of age simulated with the Besançon Galaxy model (Robin et al. 2003) in the direction of the system.

\begin{tabular}{lcc}
\hline \hline Population & Age & Fraction \\
\hline Disc & $<1 \mathrm{Gyr}$ & $3 \%$ \\
& $1-3 \mathrm{Gyr}$ & $16 \%$ \\
& $3-5 \mathrm{Gyr}$ & $23 \%$ \\
& $5-7 \mathrm{Gyr}$ & $21 \%$ \\
& $7-10 \mathrm{Gyr}$ & $24 \%$ \\
Thick disc & $11 \mathrm{Gyr}$ & $13 \%$ \\
Halo & $14 \mathrm{Gyr}$ & $0 \%$ \\
\hline
\end{tabular}

Notes. The distance of simulated stars ranges from $60 \mathrm{pc}$ to $120 \mathrm{pc}$, their proper motion $\mu_{\alpha}$ from -230 mas $\mathrm{yr}^{-1}$ to -140 mas $\mathrm{yr}^{-1}$

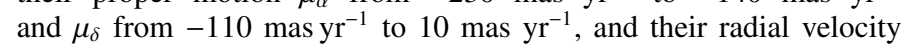
from $-30 \mathrm{~km} \mathrm{~s}^{-1}$ to $30 \mathrm{~km} \mathrm{~s}^{-1}$.

type M3.5/M4.5; Reid \& Cruz 2002 discuss this discontinuity in detail). This absolute magnitude range is consistent with $M_{J}=9.60$ for Baraffe et al. (1998) evolution models with the stellar parameters estimated in the sections below $(3.3,3.4$, and 3.5). This translates into a distance of 67 to $117 \mathrm{pc}$.

We estimate the distance to the T dwarf with the spectral type vs $M_{J}$ relation derived by Dupuy \& Liu (2012) from brown dwarfs with a measured trigonometric parallax. The absolute magnitude of a field T2 dwarf is $M_{J}=14.9 \pm 0.5$, translating into a distance of between 51 to $83 \mathrm{pc}$.

The components are separated by $7.7^{\prime \prime}$. At the distance of the system, this translates to a projected separation of 393 to $901 \mathrm{AU}$.

\subsection{Kinematics and age}

The proper motion translates into a rather high tangential velocity $\left(77 \pm 30 \mathrm{~km} \mathrm{~s}^{-1} \mathrm{yr}^{-1}\right)$, suggesting an old age. The M-dwarf spectrum shows no obvious Doppler shift. At the resolution of $\mathrm{X}$-shooter, this constrains the radial velocity to an interval of -30 to $30 \mathrm{~km} \mathrm{~s}^{-1}$. From these kinematics, simulations with the Besançon Galactic population model (Robin et al. 2003) found a probability of $81 \%$ that the age is older than $3 \mathrm{Gyr}$ and a probability of $58 \%$ that it is older than $5 \mathrm{Gyr}$ (see Table 5). The older age derived from the kinematics is independently corroborated by the absence of $\mathrm{H} \alpha$ emission in the optical spectrum of the primary. According to West et al. (2008), such a low activity level for a mid-M dwarf indicates an age $\gtrsim 6 \mathrm{Gyr}$.

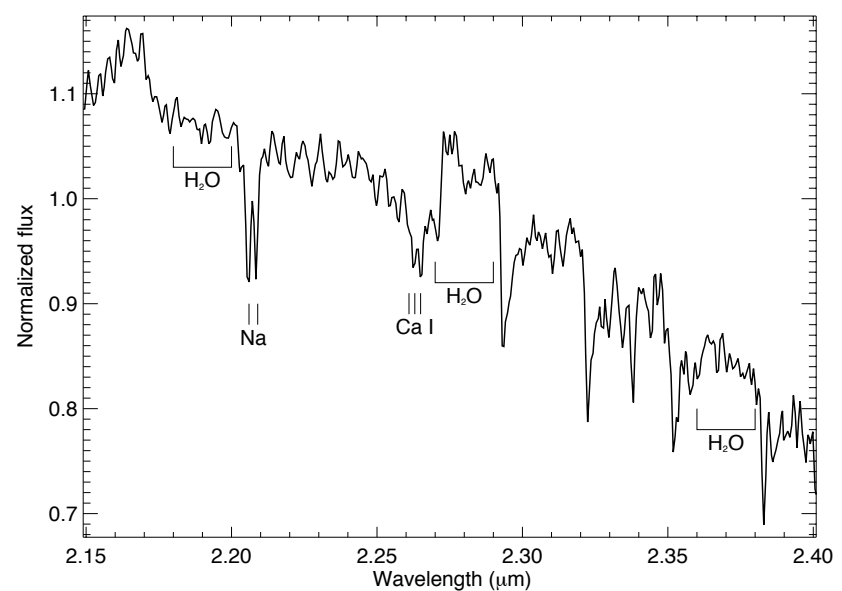

Fig. 4. $K$-band spectrum of 2MASS 1118 obtained with X-shooter at VLT. The features used to compute the metallicity are highlighted.

\subsection{Metallicity}

The metallicity of the system can be derived from the $K$-band spectrum of the primary. As shown by Rojas-Ayala et al. (2010), the metallicity of $\mathrm{M}$ dwarfs can be inferred from the strength of their $\mathrm{Na}$ I $(2.2 \mu \mathrm{m}), \mathrm{Ca} \mathrm{I}(2.26 \mu \mathrm{m})$ features, together with the $\mathrm{H}_{2} \mathrm{O}-\mathrm{K}$ index defined by Covey et al. (2010). These features are highlighted in Fig. 4.

From the Rojas-Ayala et al. (2010) and Terrien et al. (2012) relations, the metallicity is $[\mathrm{Fe} / \mathrm{H}]=-0.1 \pm 0.1$ and is consistent with the average metallicity in the solar neighbourhood.

\subsection{Effective temperature, gravity, and mass}

We compared the most recent version of the BT-Settl stellar atmosphere models (Allard et al. 2012, 2013) with the observed spectra of the two components. These models are computed with the PHOENIX code and take into account i) the solar abundances revised by Caffau et al. (2011); ii) the most recent BT2 version of the water-vapor line lists by Barber et al. (2008); iii) slightly revised atomic and molecular opacities; iv) a cloud model based on condensation and sedimentation timescales by Rossow (1978), supersaturation computed from pre-tabulated chemical equilibrium, and mixing from 2D radiation hydrodynamic simulations by Freytag et al. (2010). The models are available on-line ${ }^{3}$ and are fully described in Allard et al. (2012), Rajpurohit et al. (2012), and Allard et al. (2013).

The M-dwarf spectrum is best fitted by a synthetic spectrum with $T_{\text {eff }}=3000 \mathrm{~K}, \log g=5.0$ (Fig. 3). The inferred temperature is consistent with the relation between effective temperature scale and spectral subtype for $M$ dwarfs (see e.g. Fig. 5 in Rajpurohit et al. 2013). The Baraffe et al. (1998) evolution models give $\log g=5.2$ (and a mass of 0.10 to $0.15 M_{\odot}$ ) for an age of $6 \mathrm{Gyr}$ and $T_{\text {eff }}=3000 \pm 100 \mathrm{~K}$, which again is reassuringly consistent with the value inferred from the spectrum.

Figure 5 compares the spectrum of the T dwarf with models of varying effective temperature and gravity. We fixed the metallicity of the models to solar, using our measurement from the near-infrared spectrum of the M dwarf to remove one degree of freedom. A scaling factor was applied to normalize the median flux in the range $1.2-1.3 \mu \mathrm{m}$ to unity. The best fit, based on visual inspection of Fig. 5 , is obtained for $T_{\text {eff }}=1300 \mathrm{~K}$ and $\log g=5.0$. However, one must be aware that the models

3 http://phoenix.ens-lyon.fr/simulator 


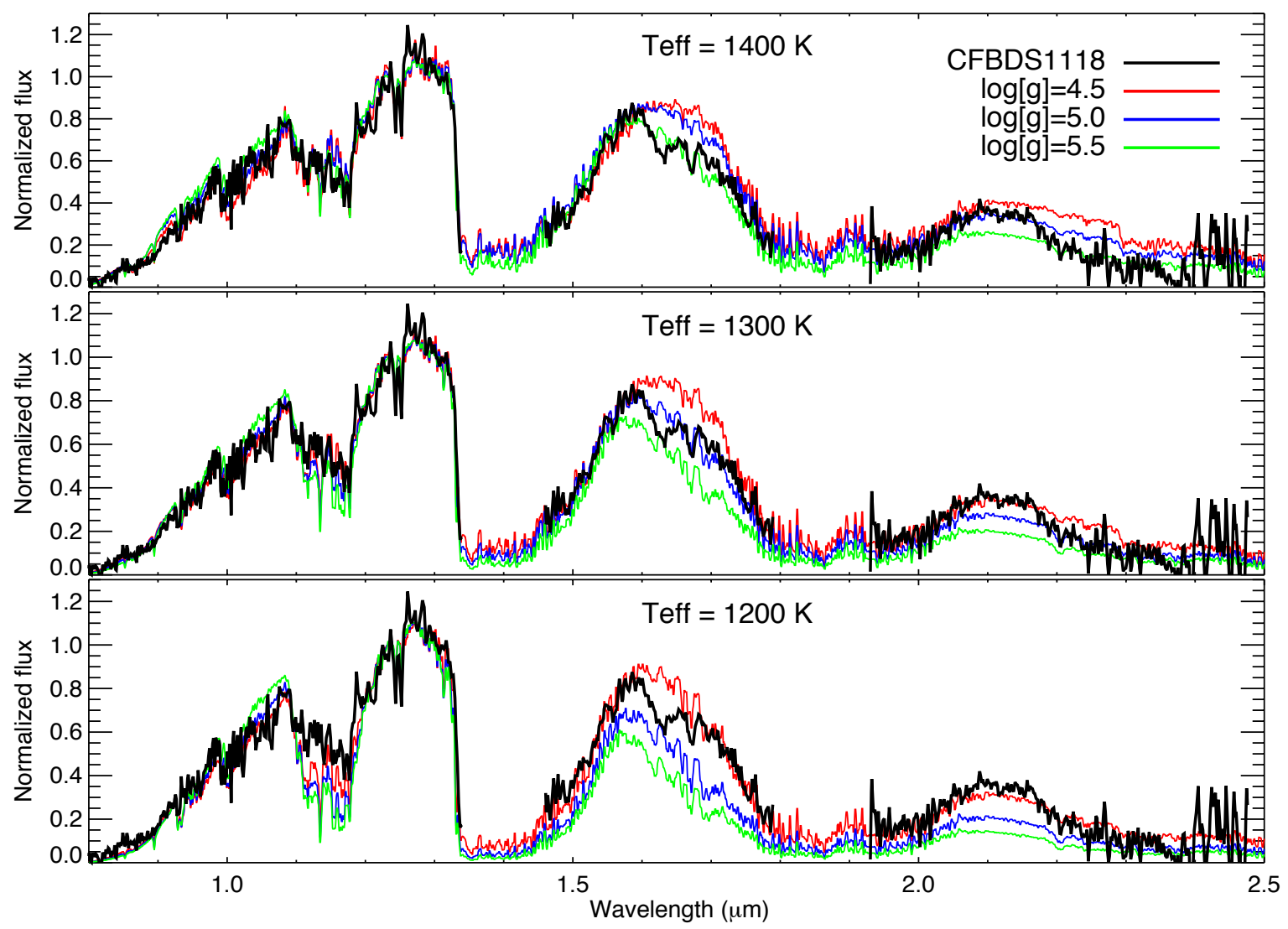

Fig. 5. Comparison of CFBDS 1118 spectrum with BT-Settl models of varying effective temperature and gravity. A scaling factor is applied to normalize the median flux in the range $1.2-1.3 \mu \mathrm{m}$ to unity. The blue line in the middle panel shows the best-fitting model $\left(T_{\text {eff }}=1300 \mathrm{~K}\right.$ and $\log g=5.0)$.

Table 6. Physical parameters derived from the Baraffe et al. (2003) evolution models.

\begin{tabular}{lccccc}
\hline \hline $\begin{array}{l}\text { Age } \\
(\mathrm{Gyr})\end{array}$ & $\begin{array}{c}\text { Mass } \\
\left(M_{\odot}\right)\end{array}$ & $\begin{array}{c}T_{\text {eff }} \\
(\mathrm{K})\end{array}$ & $\log g$ & $\begin{array}{c}\log L \\
\left(L_{\odot}\right)\end{array}$ & $\begin{array}{c}\text { Radius } \\
\left(R_{\odot}\right)\end{array}$ \\
\hline 0.5 & 0.03 & 1264 & 4.9 & -4.6 & 0.1 \\
1 & 0.04 & 1271 & 5.1 & -4.7 & 0.09 \\
5 & 0.06 & 1120 & 5.4 & -5.1 & 0.08 \\
5 & 0.07 & 1524 & 5.5 & -4.5 & 0.08 \\
10 & 0.07 & 1289 & 5.5 & -4.8 & 0.08 \\
\hline
\end{tabular}

Notes. The listed temperatures are the closest model grid points to our determination $T_{\text {eff }}=1300 \pm 100 \mathrm{~K}$.

still miss some opacity in the $H$ band. Thus the $1200 \mathrm{~K} ; 4.5$ dex and $1400 \mathrm{~K} ; 5.0$ dex solutions cannot be excluded.

Table 6 compares these values with those obtained from the Baraffe et al. (2003) evolution models for a $T_{\text {eff }}=1300 \pm 100 \mathrm{~K}$ and for a range of ages. For the age range we adopted, the gravity value derived from the model atmosphere fit to the spectrum is inconsistent with that obtained from the evolution models at the fitted effective temperature. This could potentially be due to (i) an erroneous age estimate or (ii) the known limitations of the cloud model, which is the largest source of uncertainty in brown-dwarf atmosphere models. For example, the dust opacity might be poorly estimated due to an incomplete treatment of grain growth mechanisms, which are being addressed in an upcoming revision of the models. The evidence that the system is old, from the low magnetic-activity level in the $\mathrm{M}$ dwarf and the kinematics of the system, is strong. This leaves (ii) as the most likely explanation.
We therefore adopted the gravity range obtained from the evolution models at old ages, $\log g=5.4$ at $5 \mathrm{Gyr}$, and 5.5 at $10 \mathrm{Gyr}$. This translates into a mass of 0.06 to $0.07 M_{\odot}$ for CFBDS 1118, for ages of $5 \mathrm{Gyr}$ and $10 \mathrm{Gyr}$ and a luminosity $\log L / L_{\odot}=-4.80 \pm 0.15$.

\section{Discussion}

Figure 6 shows the colour versus spectral type diagram for L and $\mathrm{T}$ dwarfs with $J-K$ photometry measured to better than 0.25 mag and a near-infrared spectral type, as compiled by Dupuy \& Liu (2012). The overall trend is a mild reddening of $J-K$ along the L-dwarf sequence, followed by its blueing along the $\mathrm{T}$ dwarf sequence. This behaviour reflects the evolution of dust grains along the L-dwarf sequence and their settling below the photosphere of T dwarfs, as well as dispersion of the clouds and possibly other unknown mechanisms. The diagram shows broad scatter at any given spectral type, most likely arising from differences in metallicity, gravity, and detailed dust clouds properties. Redder colours can result from low gravity, high metallicity, more dust opacity, or a combination of these factors.

The diagram highlights CFBDS 1118, as well as HN Peg B. The latter is a very young T2.5 \pm 0.5 dwarf, with an age of just $0.3 \pm 0.2 \mathrm{Gyr}$ estimated from its G0 main-sequence primary. Its estimated mass is $0.021 \pm 0.009 M_{\odot}$, its effective temperature $1130 \pm 70 \mathrm{~K}$, and its gravity 4.8 (Luhman et al. 2007; Leggett et al. 2008). The metallicity of the system is $-0.01 \pm 0.03$ (Valenti \& Fischer 2005). These two T dwarfs therefore differ widely on age, mass, and gravity, and slightly on effective temperature. 


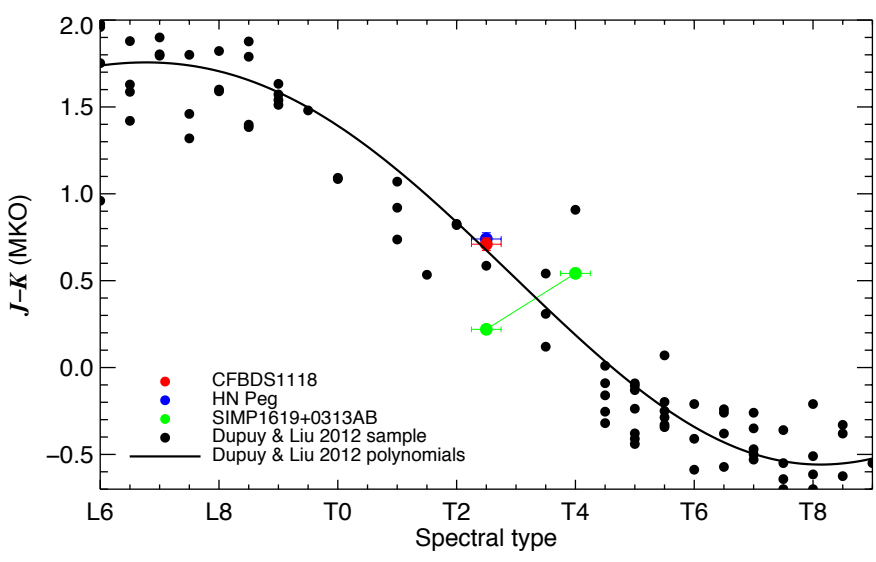

Fig. 6. $J-K(\mathrm{MKO})$ colour versus spectral type for $\mathrm{L}$ and $\mathrm{T}$ dwarfs. The red and blue markers highlight CFBDS 1118 and HN Peg B, respectively. The green symbols mark the two components of the SIMP 1619+0313AB binary.

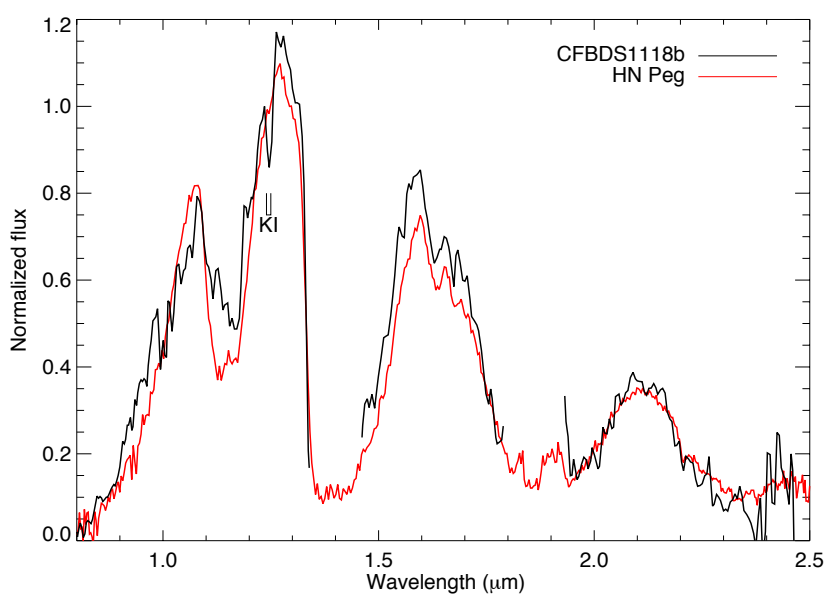

Fig. 7. Spectrum of CFBDS 1118 (black) obtained with X-shooter, binned to the resolution of the Luhman et al. (2007) spectrum of HN Peg B (red) obtained with SpeX at the NASA Infrared Telescope Facility. A scaling factor is applied to normalize the median flux in the range $1.2-1.3 \mu \mathrm{m}$ to unity. The $1.25-1.26 \mu \mathrm{m}$ potassium doublet is highlighted.

Despite their contrasting ages and gravity, CFBDS 1118 and HN Peg B have very similar $J-K$ colours, 0.71 and 0.74 . Gravity therefore contributes very little to the $J-K$ scatter of early-T dwarfs, or its effect is compensated for by other factors. As the $\mathrm{L} / \mathrm{T}$ transition marks cloud-clearing, detailed dust properties and cloud coverage may play an important role, as illustrated by the SIMP J1619+0313AB T dwarf binary (Artigau et al. 2011). That system (also shown in Fig. 6) has unexpectedly reversed colours, with a bluer $J-K$ for the earlier-type (T2.5) primary than for the later-type (T4) secondary. The common age and metallicity of the two components leaves contrasting cloud properties, by elimination, as the preferred explanation for the colour reversal (Artigau et al. 2011).

Figure 7 compares the near-infrared spectra of CFBDS 1118 and HN Peg B (from Luhman et al. 2007). The two spectra are extremely similar, with their potassium doublet feature as the main difference. While the young, low-gravity, HN Peg B shows no discernible $\mathrm{K} \mathrm{I}$ lines, that feature is readily apparent in the CFBDS 1118 spectrum, as expected from its higher gravity (and older age). The increasing strength of $\mathrm{K}$ I lines

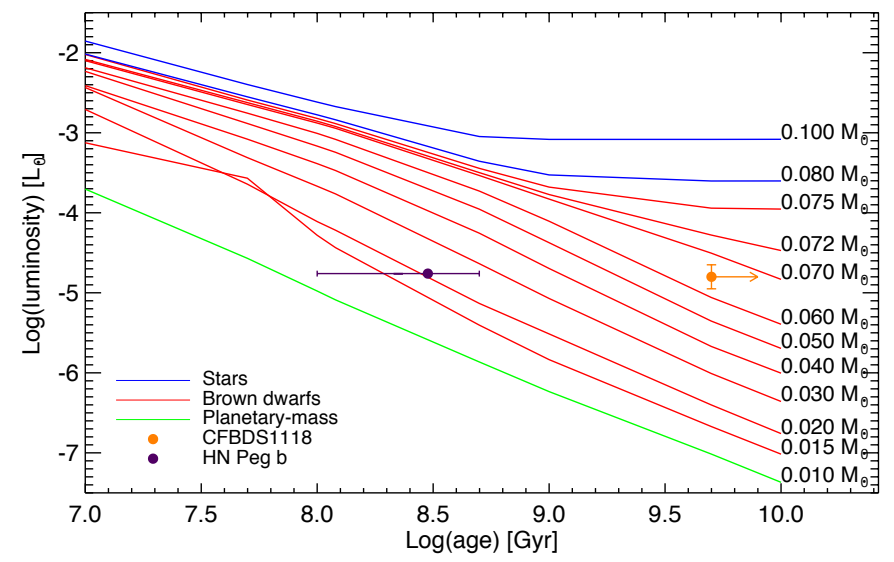

Fig. 8. Luminosity of solar-metallicity $M$ dwarfs and substellar objects against time after formation according to Baraffe et al. (2003). The black dot marks HN Peg B and the orange dot shows CFBDS 1118. The luminosity of HN Peg B is $\log L / L_{\odot}=-4.76$, with an error bar of 0.02 (Leggett et al. 2008), lower than the symbol size.

with increasing gravity has been extensively studied in $\mathrm{L}$ dwarfs (Allers \& Liu 2013), but that trend (mildly) reverses for late-T dwarfs. They have slightly stronger $\mathrm{K} I$ lines at lower gravity, because the $\mathrm{CH}_{4}$ and $\mathrm{H}_{2} \mathrm{O}$ bands that define their pseudocontinuum for the potassium doublet are slightly more sensitive to pressure-broadening than is the atomic feature (Allard et al. 2012; Delorme et al. 2012). The two spectra have very similar (normalized) $K$-band flux, in spite of very contrasting ages and gravities. This constrasts with the late-T dwarfs, where high gravity enhances collision-induced absorption by $\mathrm{H}_{2}$ and, for a fixed effective temperature therefore suppresses the $K$-band flux with respect to the other bands (e.g. Knapp et al. 2004; Delorme et al. 2012). The $J-K$ colour at the $\mathrm{L} / \mathrm{T}$ transition is therefore controlled by some other parameter, which most likely is dustor cloud-related.

Figure 8 shows luminosity against time for different masses for Baraffe et al. (2003) models. The orange dot marks the position of CFBDS 1118. The masses of old ( $>5 \mathrm{Gyr}$ ) late-L and early-T dwarfs are much better constrained for a given uncertainty on their luminosity than those of most substellar objects. At these ages, objects below $\sim 60$ Jupiter masses have cooled into late-Ts and cooler, while objects above $\sim 78$ Jupiter masses remain on the bottom of the main sequence and are at most mid-Ls. As a result, old age and an L-T transition spectral type alone constrain the mass to $\pm 15 \%$. Adding an even a moderately precise luminosity and an age pinpoints the mass, within the possible systematic errors in the theoretical model grid. As an example, a $20 \%$ uncertainty on an $L=10^{-4.5} L_{\odot}$ luminosity translates into a $3 \%$ fractional mass uncertainty at $10 \mathrm{Gyr}\left(74.9 \pm 2.3 M_{\text {Jup }}\right)$, and into a $13 \%$ uncertainty at $0.5 \mathrm{Gyr}\left(36 \pm 5.6 M_{\mathrm{Jup}}\right)$. Given our confidence in its old age, the discrepancies in the physical parameters determined from evolution and atmosphere models of CFBDS 1118 are well established and make an interesting testbed.

\section{Summary}

Brown-dwarfs companions to main-sequence stars provide unique benchmarks for studies of cool atmospheres, because the better-understood primary star provides the age, distance, and metallicity of the substellar companion. These systems therefore 
provide unique tests of cool-atmosphere models by vastly reducing the number of degrees of freedom.

We identified a $7.7^{\prime \prime}$-separation common proper-motion M-dwarf and T-dwarf binary within our CFBD survey (Delorme et al. 2008b). The red colours of the companion identify it as an early-T dwarf, as confirmed by its spectroscopic follow-up. The high tangential velocity of the system and the low upper limit on the $\mathrm{H}_{\alpha}$ feature in the spectrum of the M4.5-M5 primary indicate that the system is old ( $\gtrsim 5 \mathrm{Gyr}$ ). Observations of the two components with the wide spectral coverage of X-shooter constrained the physical parameters of the system.

The T dwarf, with its metallicity fixed to $[\mathrm{Fe} / \mathrm{H}]=-0.1 \pm 0.1$ from the $\mathrm{M}$ dwarf and a well-constrained mass estimate, provides a valuable anchor. Thanks to its older age, its properties depend only weakly on its exact age: since older brown dwarfs cool down and contract very slowly (the $T_{\text {eff }}$ of a $70 M_{\text {Jup }}$ object, for instance, only drops from $1520 \mathrm{~K}$ to $1290 \mathrm{~K}$ between 5 Gyr and $10 \mathrm{Gyr}$, and its $\log g$ increases almost insignificantly from 5.47 to 5.50), they are much less affected by the age, mass, and luminosity degeneracy that generally hinders field brown-dwarf studies. The T dwarf also lies close to the boundary between the lowest mass stars (whose luminosity remains constant) and brown dwarfs (with decreasing luminosity). It is one of the most massive brown dwarfs, just below the hydrogenburning limit.

The early-T spectral type of the brown dwarf enhances the value of the system, because the cloud-clearing that occurs at the $\mathrm{L} / \mathrm{T}$ transition is very sensitive to gravity and metallicity (e.g. Oppenheimer et al. 2013; Allers \& Liu 2013). This produces large scatter in the colours, with low-gravity objects - such as the late-L-type planet 2M1207b (Chauvin et al. 2004) - usually having redder $J-K$ colours. Comparison with HN Peg B, a much younger T2.5 dwarf companion to a main-sequence star, however, found that they have very similar photometric and spectroscopic properties. By contrast, the photometric properties of the two early-T components of the SIMP 1619+0313AB system showed that they had very different dust-dissipation histories in spite of being coeval objects. This suggests that the dust content of the atmosphere, which appears to be responsible for most of the scatter in L/T transition objects, is not simply controlled by gravity and metallicity: though dust content generally increases with lower gravity and higher metallicity, several exceptions shows that additional parameters are important.

Acknowledgements. We would like the thank the referee, whose comments helped improve our manuscript. We acknowledge observing support by the CFHT and ESO staffs, and thank the CFHT executive director for granting us discretionary observing time. We acknowledge financial support from "Programme National de Physique Stellaire" (PNPS) of CNRS/INSU, France.

\section{References}

Allard, F., Hauschildt, P. H., Alexander, D. R., Tamanai, A., \& Schweitzer, A. 2001, ApJ, 556, 357

Allard, F., Homeier, D., \& Freytag, B. 2012, Roy. Soc. London Philos. Trans. Ser. A, 370, 2765

Allard, F., Homeier, D., Freytag, B., et al. 2013, Mem. Soc. Astron. Ital. Suppl., 24,128

Allers, K. N., \& Liu, M. C. 2013, ApJ, 772, 79

Artigau, É., Bouchard, S., Doyon, R., \& Lafrenière, D. 2009, ApJ, 701, 1534

Artigau, É., Lafrenière, D., Doyon, R., et al. 2011, ApJ, 739, 48

Baraffe, I., Chabrier, G., Allard, F., \& Hauschildt, P. H. 1998, A\&A, 337, 403

Baraffe, I., Chabrier, G., Barman, T. S., Allard, F., \& Hauschildt, P. H. 2003, A\&A, 402, 701

Bate, M. R., Bonnell, I. A., \& Bromm, V. 2003, MNRAS, 339, 577

Bertin, E. 2006, in Astronomical Data Analysis Software and Systems XV, eds.

C. Gabriel, C. Arviset, D. Ponz, \& S. Enrique, ASP Conf. Ser., 351, 112
Bertin, E., \& Arnouts, S. 1996, A\&AS, 117, 393

Biller, B. A., Kasper, M., Close, L. M., Brandner, W., \& Kellner, S. 2006, ApJ, 641, L141

Blake, C. H., Charbonneau, D., White, R. J., et al. 2008, ApJ, 678, L125 Boulade, O., Charlot, X., Abbon, P., et al. 2003, in Proc. SPIE, 4841, 72 Burgasser, A. J., Kirkpatrick, J. D., Cutri, R. M., et al. 2000, ApJ, 531, L57 Burgasser, A. J., Kirkpatrick, J. D., \& Lowrance, P. J. 2005, AJ, 129, 2849 Burgasser, A. J., Geballe, T. R., Leggett, S. K., Kirkpatrick, J. D., \& Golimowski, D. A. 2006, ApJ, 637, 1067

Burgasser, A. J., Liu, M. C., Ireland, M. J., Cruz, K. L., \& Dupuy, T. J. 2008, ApJ, 681, 579

Burgasser, A. J., Simcoe, R. A., Bochanski, J. J., et al. 2010, ApJ, 725, 1405 Burgasser, A. J., Luk, C., Dhital, S., et al. 2012, ApJ, 757, 110

Burningham, B., Pinfield, D. J., Leggett, S. K., et al. 2009, MNRAS, 395, 1237 Burningham, B., Cardoso, C. V., Smith, L., et al. 2013, MNRAS, 433, 457 Caffau, E., Ludwig, H.-G., Steffen, M., Freytag, B., \& Bonifacio, P. 2011, Sol. Phys., 268, 255

Chauvin, G., Lagrange, A.-M., Dumas, C., et al. 2004, A\&A, 425, L29 Deacon, N. R., Liu, M. C., Magnier, E. A., et al. 2012a, ApJ, 757, 100 Deacon, N. R., Liu, M. C., Magnier, E. A., et al. 2012b, ApJ, 755, 94 Delorme, P., Delfosse, X., Albert, L., et al. 2008a, A\&A, 482, 961 Delorme, P., Willott, C. J., Forveille, T., et al. 2008b, A\&A, 484, 469 Delorme, P., Albert, L., Forveille, T., et al. 2010, A\&A, 518, A39 Delorme, P., Gagné, J., Malo, L., et al. 2012, A\&A, 548, A26 Devillard, N. 1997, The Messenger, 87, 19

Dupuy, T. J., \& Liu, M. C. 2012, ApJS, 201, 19

Faherty, J. K., Burgasser, A. J., West, A. A., et al. 2010, AJ, 139, 176

Freytag, B., Allard, F., Ludwig, H., Homeier, D., \& Steffen, M. 2010, A\&A, 513, A19

Goldman, B., Marsat, S., Henning, T., Clemens, C., \& Greiner, J. 2010, MNRAS, 405, 1140

Golimowski, D. A., Leggett, S. K., Marley, M. S., et al. 2004, AJ, 127, 3516

Helling, C., Ackerman, A., Allard, F., et al. 2008, MNRAS, 391, 1854

Kirkpatrick, J. D., Reid, I. N., Liebert, J., et al. 2000, AJ, 120, 447

Knapp, G. R., Leggett, S. K., Fan, X., et al. 2004, AJ, 127, 3553

Leggett, S. K., Saumon, D., Albert, L., et al. 2008, ApJ, 682, 1256

Loutrel, N. P., Luhman, K. L., Lowrance, P. J., \& Bochanski, J. J. 2011, ApJ, 739, 81

Luhman, K. L., Patten, B. M., Marengo, M., et al. 2007, ApJ, 654, 570

McLean, I. S., Prato, L., McGovern, M. R., et al. 2007, ApJ, 658, 1217

Modigliani, A., Goldoni, P., Royer, F., et al. 2010, in SPIE Conf. Ser., 7737, 773728

Moorwood, A., Cuby, J.-G., \& Lidman, C. 1998, The Messenger, 91,9

Morley, C. V., Fortney, J. J., Marley, M. S., et al. 2012, ApJ, 756, 172

Mugrauer, M., Seifahrt, A., Neuhäuser, R., \& Mazeh, T. 2006, MNRAS, 373, L31

Nakajima, T., Oppenheimer, B. R., Kulkarni, S. R., et al. 1995, Nature, 378, 463 Oppenheimer, B. R., Baranec, C., Beichman, C., et al. 2013, ApJ, 768, 24

Puget, P., Stadler, E., Doyon, R., et al. 2004, in SPIE Conf. Ser. 5492, eds. A. F. M. Moorwood, \& M. Iye, 978

Radigan, J., Jayawardhana, R., Lafrenière, D., et al. 2012, ApJ, 750, 105 Rajpurohit, A. S., Reylé, C., Schultheis, M., et al. 2012, A\&A, 545, A85 Rajpurohit, A. S., Reylé, C., Allard, F., et al. 2013, A\&A, 556, A15

Reid, I. N., \& Cruz, K. L. 2002, AJ, 123, 2806

Reid, I. N., Hawley, S. L., \& Gizis, J. E. 1995, AJ, 110, 1838

Reipurth, B., \& Clarke, C. 2001, AJ, 122, 432

Reylé, C., Scholz, R.-D., Schultheis, M., Robin, A. C., \& Irwin, M. 2006, MNRAS, 373, 705

Reylé, C., Delorme, P., Willott, C. J., et al. 2010, A\&A, 522, A112

Robin, A. C., Reylé, C., Derrière, S., \& Picaud, S. 2003, A\&A, 409, 523

Rojas-Ayala, B., Covey, K. R., Muirhead, P. S., \& Lloyd, J. P. 2010, ApJ, 720, L113

Saumon, D., Marley, M. S., Cushing, M. C., et al. 2006, ApJ, 647, 552

Scholz, R.-D. 2010, A\&A, 515, A92

Scholz, R.-D., McCaughrean, M. J., Lodieu, N., \& Kuhlbrodt, B. 2003, A\&A, 398, L29

Skrutskie, M. F., Cutri, R. M., Stiening, R., et al. 2006, AJ, 131, 1163

Stamatellos, D., \& Whitworth, A. P. 2009, MNRAS, 400, 1563

Terrien, R. C., Mahadevan, S., Bender, C. F., et al. 2012, ApJ, 747, L38

Thalmann, C., Carson, J., Janson, M., et al. 2009, ApJ, 707, L123

Valenti, J. A., \& Fischer, D. A. 2005, ApJS, 159, 141

Vernet, J., Dekker, H., D'Odorico, S., et al. 2011, A\&A, 536, A105

Vrba, F. J., Henden, A. A., Luginbuhl, C. B., et al. 2004, AJ, 127, 2948

West, A. A., Hawley, S. L., Bochanski, J. J., et al. 2008, AJ, 135, 785

Wilson, J. C., Kirkpatrick, J. D., Gizis, J. E., et al. 2001, AJ, 122, 1989

Zacharias, N., Monet, D. G., Levine, S. E., et al. 2004, BAAS, 36, 1418 